\title{
Theoretical and Experimental Study of Split Semi Horse Shoe Structure
}

\author{
Ghanshyam Singh $^{1^{*}}$, Shyam S. Pattnaik ${ }^{2}$ \\ ${ }^{1}$ Dept. of Electronics \& Communication Engineering, Feroze Gandhi Institute of Engineering \& Technology, Raebareli, India. \\ ${ }^{2}$ Director, National Institute of Technical Teachers Training and Research, Chandigarh, India. \\ *corresponding author, E-mail: ghanshyamtanu@rediffmail.com
}

\begin{abstract}
In this paper a new planar metamaterial structure that looks like semi-horse shoe in shape (SSHSS) is designed and simulated. Theoretical analysis of proposed structure done using equivalent circuit theory. Medium effective parameters are extracted using modified NRW approach which proved the metamaterial property of the new unit cell. Radiation pattern, directivity and gain of the new structure were illustrated which gives the possibility of using SSHSS as the antenna. Proposed structure shows multiband characteristics. This antenna shows high directivity $(7.92 \mathrm{dBi}, 7.86 \mathrm{dBi}, 10.11 \mathrm{dBi})$ and moderate gain $(2.55 \mathrm{dBi}, 3.90 \mathrm{dBi}, 5.07 \mathrm{dBi})$ at $5.83 \mathrm{GHz}, 8.41 \mathrm{GHz}$, $10.68 \mathrm{GHz}$ respectively. RT duroid is used for fabrication of prototype of the proposed structure. This new structure can be used as metamaterial inspired antenna as well as normal patch antenna. Experimental results shows good agreement with simulated and theoretical results. The proposed structure has been simulated using IE3D electromagnetic simulator.
\end{abstract}

\section{Introduction}

Artificial Metamaterials were first proposed by Prof. Veselago in 1968[1]. Present day communication technology demands miniaturized wireless systems. Antenna designing is very challenging domain for advanced communication devices. In early decade of metamaterial discovery researcher focuses on realization of these periodic structures. Now they are looking for use of these artificial materials in present day communication devices. Multiband operation, pattern \& frequency reconfiguration is possible by loading of these new structures to conventional patch antennas. A large variety of Split ring Resonator (SRR) design were designed so far after first unit cell proposed by J. B. Pendry in 1999[2]. D. R. Smith et.al. in [3] have demonstrated first composite medium composed of SRR and thin wires. In [4] R.W. Ziolkowski et al. showed that the radiation power of small antennas increases by incorporation of metamaterials. Gain of patch antenna improved by using CSRR in ground plane in [5]. M.S. Sharawi et. al. in [6] proposed a new compact antenna for MIMO having good isolation between elements using CSRR in ground plane. J.
G. Joshi et. al. proposed offset fed diamond shaped split ring (DSSR) structure to improved bandwidth [7]. In [8] Vipul sharma et. al. presents a new planar microstrip elliptical split-ring resonator (ESRR) showing negative refractive index at multiple bands .In [9] a new planar triangular split ring resonator (TSR) showing left hand property at $\mathrm{Ku}$ band is proposed. In [10] G. Singh et. al. proposed a new planar split semi horse shoe structure (SSHSS) used as antenna for $\mathrm{X}$ band. To change the resonance frequency from $\mathrm{X}$ band to ISM band, split vertical length \& stub length has been changed in present work. Every shape of SRR has its own advantages and disadvantages. This new structure does not incorporate metallic rod for getting negative permittivity.

This paper is organized in five sections. Design specifications and theoretical analysis of the new structure are presented in section II. Simulation and use of simulation results to verify the metamaterial property is presented in section III. Experimental findings are presented in section IV. Section V showed the conclusion of paper.

\section{Geometry of Proposed Resonator}

In this section theoretical analysis using equivalent circuit theory and physical specifications of split semi-horse shoe structure (SSHSS) is presented. The physical specifications of proposed structure are given in Table 1. RT Duroid 5880 substrate having relative permittivity 2.20, loss tangent $=0.0009$ and thickness $\mathrm{h}=1.575 \mathrm{~mm}$ is used to simulate and fabricate the prototype. Simulation of the structure is done by IE3D electromagnetic simulator software.

Table1: Physical dimension of the proposed structure

\begin{tabular}{|c|c|c|c|c|c|c|c|c|c|}
\hline Parameters & $\mathrm{r}_{1}$ & $\mathrm{r}_{2}$ & $\mathrm{r}_{3}$ & $\mathrm{r}_{4}$ & $\mathrm{~W}$ & $\mathrm{~S}$ & $\mathrm{w}$ & $\mathrm{L}$ & $\mathrm{D}$ \\
\hline $\begin{array}{l}\text { Present } \\
\text { Design } \\
\text { Values }\end{array}$ & 6 & 8 & 9 & 11 & 2 & 1 & 4.4 & 24.16 & 2 \\
\hline Ref.[10] & 6 & 8 & 9 & 11 & 2 & 1 & 3.6 & 20.59 & 2 \\
\hline Ref.[11] & 6 & 8 & 9 & 11 & 2 & 1 & 4 & 23.47 & 2 \\
\hline
\end{tabular}

Figure 1 shows the geometry of proposed split semi-horse shoe structure with both rings has vertical split cut in $\mathrm{Y}$ axis. 


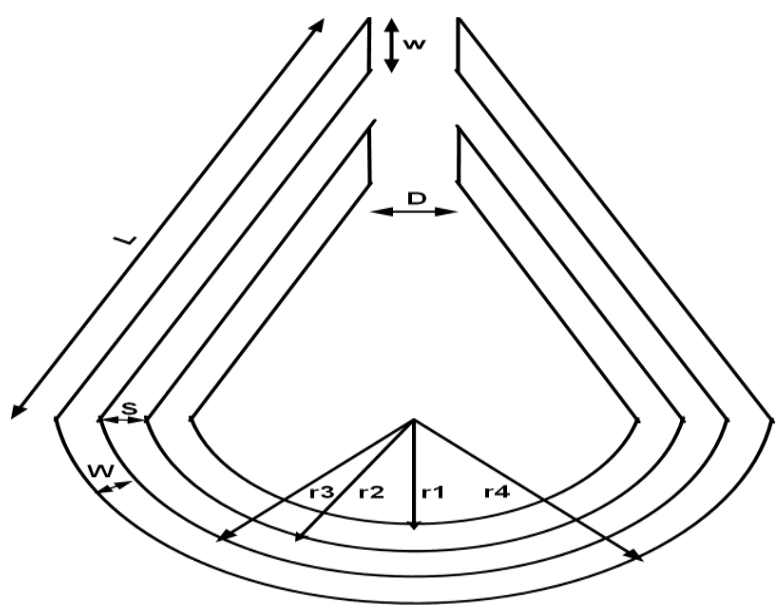

Figure 1: Geometry of Split Semi-Horse Shoe Structure

\subsection{Theoretical Analysis of SSHSS}

Theoretical analysis of the proposed structure is done by principles of equivalent circuit theory. The antenna resonates due to the reactance (i.e., inductance and capacitance) present in the structure. These inductances and capacitances lead the structure to behave as a LC tank circuit. For the calculation of inductance and capacitance the of unit cell, structure is divided in four sections as shown in [12] Fig.2. Equivalent circuit model of the SSHSS is shown in Fig.3.

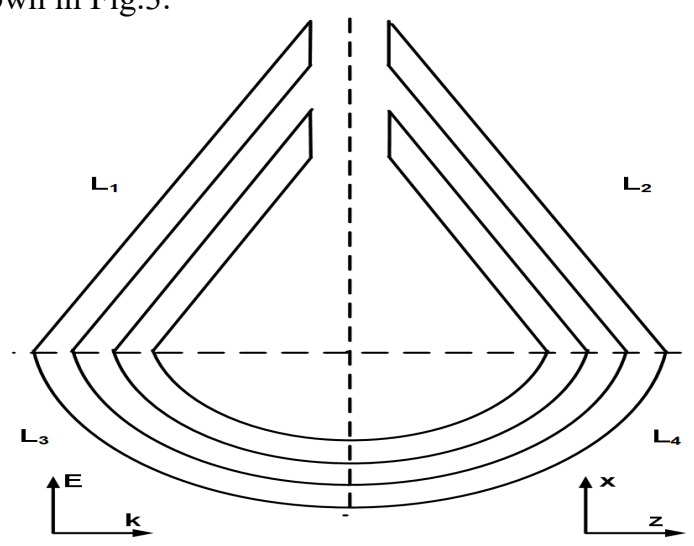

Figure 2: SSHSS divisions for calculation of inductors

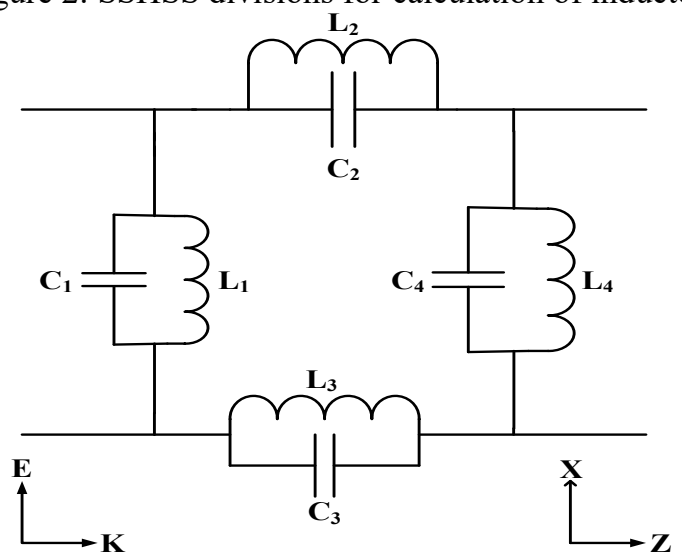

Figure 3: Equivalent circuit model of the SSHSS

$\mathrm{L}_{1}$ and $\mathrm{L}_{2}$ estimated by following expressions [13]

$$
\begin{gathered}
\mathrm{L}_{1,2}=\frac{\mathrm{K} \mu \mathrm{N}^{2} \mathrm{~L}}{2 \pi}\left[\ln \left(\frac{2}{\rho}\right)+0.5+0.178 \rho+0.0416 \rho^{2}\right]+ \\
\frac{\mu \mathrm{N}^{2} \mathrm{~L}}{2 \pi}\left[0.5 \frac{(\mathrm{N}-1) \mathrm{S}^{2}}{(\rho \mathrm{L})^{2}}+0.178 \frac{(\mathrm{N}-1) \mathrm{S}}{\mathrm{NL}}-\frac{1}{\mathrm{~N}} \ln \left(\frac{(\mathrm{W}+\mathrm{t})}{\mathrm{W}}\right)\right.
\end{gathered}
$$

where $\rho$ is the filling ratio given as $\rho=\frac{\mathrm{NW}+(\mathrm{N}-1) \mathrm{S}}{\mathrm{L}} \mathrm{N}$ is the number of rings. $\mu$ is the permeability of free space. $\mathrm{K}$ is integrality coefficient introduce to reduce the error in calculation of $\mathrm{L}$ caused by split in the strip, $\mathrm{K}$ is expressed as $\mathrm{K}=\frac{(2 \mathrm{~L}-2 \mathrm{~S})-\mathrm{D}}{(2 \mathrm{~L}-2 \mathrm{~S})}$. The self inductance of the two ring $\left(\mathrm{L}_{\text {ring }}\right)$ having width $\mathrm{W}$ and radius $\mathrm{r}$ is calculated using following equations [13];

$$
\mathrm{L}_{\text {ring }}=
$$

$\frac{\mu \mathrm{N}^{2} \mathrm{~d}}{2}\left[\ln \left(\frac{\mathrm{d}}{\mathrm{W}}\right)+0.9+0.2 \frac{\mathrm{W}^{2}}{\mathrm{~d}^{2}}\right]+\frac{\mu \mathrm{N}^{2} \mathrm{~d}}{2}\left[0.5 \frac{(\mathrm{N}-1) \mathrm{S}^{2}}{(\mathrm{\rho d})^{2}}+\right.$

$\left.0.4 \frac{(\mathrm{N}-1) \mathrm{S}(\mathrm{W}+\mathrm{S})}{\mathrm{d}^{2}}-\frac{1}{\mathrm{~N}} \ln \left(\frac{(\mathrm{W}+\mathrm{t})}{\mathrm{W}}\right)\right)$

where $\rho$ is the filling ratio given as $\rho d=N W+(N-1) S$ and $d=\frac{d_{1}+d_{2}}{2}, d_{1}=r_{1}+r_{2}, d_{2}=r_{3}+r_{4}$.

The mutual capacitance $\left(\mathrm{C}_{\text {rings }}\right)$ per unit radian between the two rings is given by equation given below [14],

$$
\mathrm{C}_{\text {rings }}=\frac{2}{\pi} \varepsilon_{0} \epsilon_{\mathrm{r}} \mathrm{rln}
$$

where $\mathrm{r}$ is radius of the ring, $\mathrm{W}$ is ring width and $\mathrm{S}$ is gap between two rings.

The mutual capacitance $\left(\mathrm{C}_{\text {strips }}\right)$ between the two strips is given by equation given below [14]:

$$
\mathrm{C}_{\text {strips }}=\frac{\varepsilon_{\mathrm{r}} \varepsilon_{0} \mathrm{AtL}}{\mathrm{S}}
$$

Where $\mathrm{A}$ is the modification factor, $\mathrm{t}$ is the thickness of metallic strip, $\mathrm{L}$ is the length of the overlapping area, $\mathrm{S}$ is the gap between strip, $\varepsilon_{\mathrm{r}}$ is the relative permittivity of the substrate $(2.20)$ and $\varepsilon_{0}$ is the permittivity of free space $\left(8.854 \times 10^{-12} \mathrm{~F} / \mathrm{m}\right)$.

The gap capacitance is estimated at the two splits. It is vital to estimate the capacitances of the outer and inner splits, but it is difficult because of the intense electromagnetic brink effects, so they are estimated by modifying the parallel plane capacitance formula with a modified factor ,that is [14];

$$
C_{\text {gap }}=\frac{\varepsilon_{\mathrm{r}} \varepsilon_{0} \mathrm{Atw}}{\mathrm{g}}
$$

Where $\mathrm{A}$ is the modification factor, $\mathrm{t}$ is the thickness of metallic strip, $w$ is the vertical width of the strip, $\varepsilon_{r}$ is the relative permittivity of the substrate $(2.20)$ and $\varepsilon_{0}$ is the permittivity of free space $\left(8.854 \times 10^{-12} \mathrm{~F} / \mathrm{m}\right)$.

The value of distributed capacitances $\mathrm{C}_{1}, \mathrm{C}_{2}, \mathrm{C}_{3}$ and $\mathrm{C} 4$ can be determined by using $\mathrm{C}_{\text {rings }}, \mathrm{C}_{\text {strips }}, \mathrm{C}_{\text {gap }}$ calculated above using the following approaches [14]:

$$
\mathrm{C}_{1}=\mathrm{C}_{2}=\mathrm{C}_{\text {strips }}+\mathrm{C}_{\text {gap }}
$$




$$
\mathrm{C}_{3}=\mathrm{C}_{4}=\frac{1}{2} \mathrm{C}_{\text {rings }}
$$

After simplifying the equivalent circuit for present design, the equivalent inductance and capacitance for first resonance frequency is found as $\mathrm{L}_{\mathrm{eq}}=36.90 \mathrm{nH}$ and $\mathrm{C}_{\mathrm{eq}}=0.0251 \mathrm{pF}$. By using the value of Leq, $\mathrm{C}_{\mathrm{eq}}$ numerically calculated resonant frequency is $\mathrm{f}_{\mathrm{r}}=5.72 \mathrm{GHz}$. While the simulated resonant frequency is $5.83 \mathrm{GHz}$ which shows the $1.88 \%$ of error and experimental frequency is $5.845 \mathrm{Ghz}$ all these three are in good agreement.

\section{Simulation Results and Discussion}

The proposed SSHSS structure is coaxially excited at $\mathrm{x}=-7.5$ $\mathrm{mm}, \mathrm{y}=-6.8 \mathrm{~mm}$ and $\mathrm{x}=2.3 \mathrm{~mm}, \mathrm{y}=10.3 \mathrm{~mm}$. Figure 4 shows the simulated reflection coefficient $\left(\mathrm{S}_{11}\right)$ and phase reversal $\left(\mathrm{S}_{12}\right)$ characteristics of the proposed metamaterial structure (SSHSS) upto $20 \mathrm{GHz}$. Figure 5, shows the reflection coefficient $\left(\mathrm{S}_{11}\right)$ at $5.83 \mathrm{GHz}$ frequency in expanded view is $-24.24 \mathrm{~dB}$, it is also clear that structure exhibits phase reversal in the resonant frequency band which implying that the wave vector changes its phase by an angle of $180^{\circ}$ at the interface. This antenna shows metamaterial behavior at 5.83 $\mathrm{GHz}, 8.41 \mathrm{GHz}, 12.67 \mathrm{GHz}, 14.87 \mathrm{GHz}$ resonance frequency while show normal behavior at $10.64 \mathrm{GHz}$. Table 2 shows the summary of the simulated results. This antenna shows multiple resonance frequency due to the multiple reactance (i.e., inductance and capacitance) present in the structure.

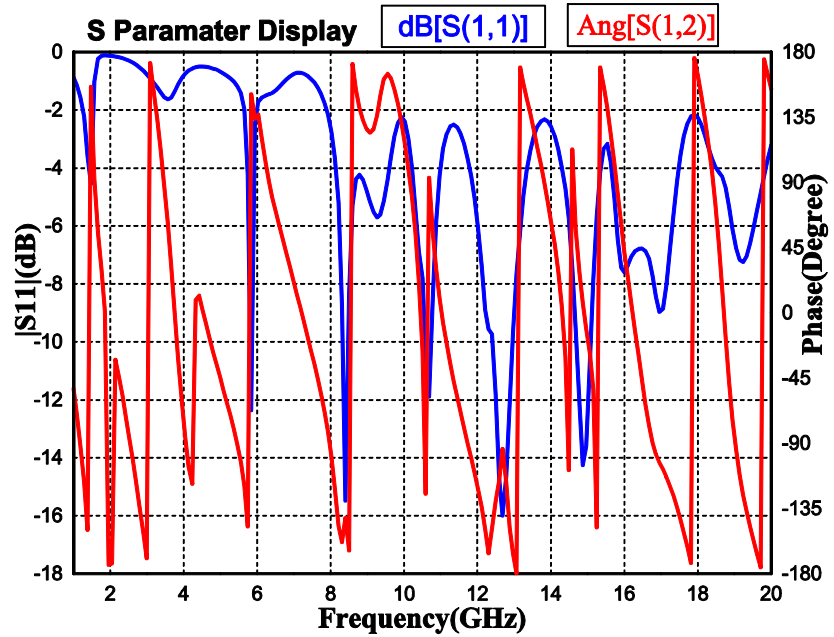

Figure 4: $\mathrm{S}_{11}(\mathrm{~dB})$ and $\mathrm{S}_{12}$ (phase angle) of the SSHSS antenna

Table 2 Summary of the simulated results

\begin{tabular}{|c|c|}
\hline $\begin{array}{c}\text { Resonance } \\
\text { Frequency }(\mathrm{GHz})\end{array}$ & Return Loss $\mathrm{S}_{11}(\mathrm{~dB})$ \\
\hline 5.83 & $\begin{array}{c}-11.50 \\
(-24.24 \mathrm{~dB} \text { expanded view })\end{array}$ \\
\hline 8.41 & -15.41 \\
\hline 10.68 & -12.04331 \\
\hline 12.67 & -15.80 \\
\hline 14.87 & -14 \\
\hline
\end{tabular}

Figure 6 depicts the simulated polar radiation pattern of the SSHSS structure in both planes.

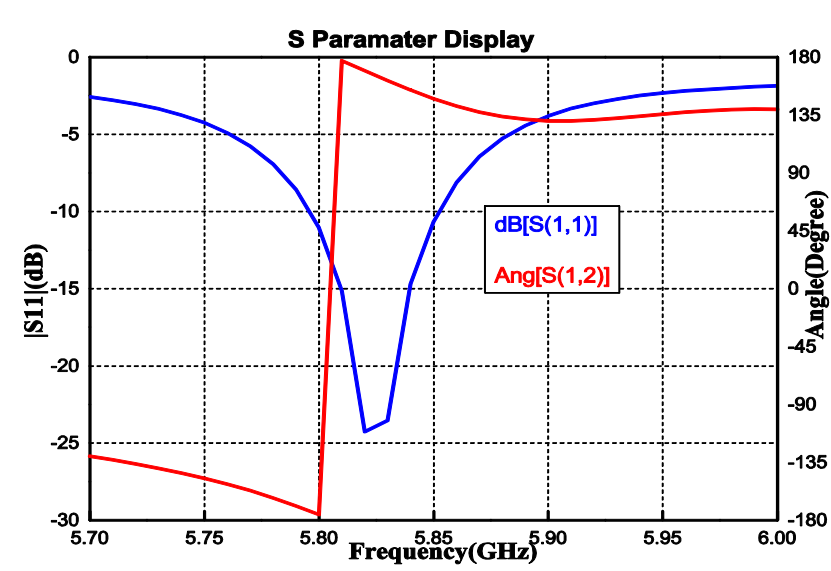

Figure 5: Expanded view of the $\mathrm{S}_{11}(\mathrm{~dB})$ and $\mathrm{S}_{12}$ (phase angle)

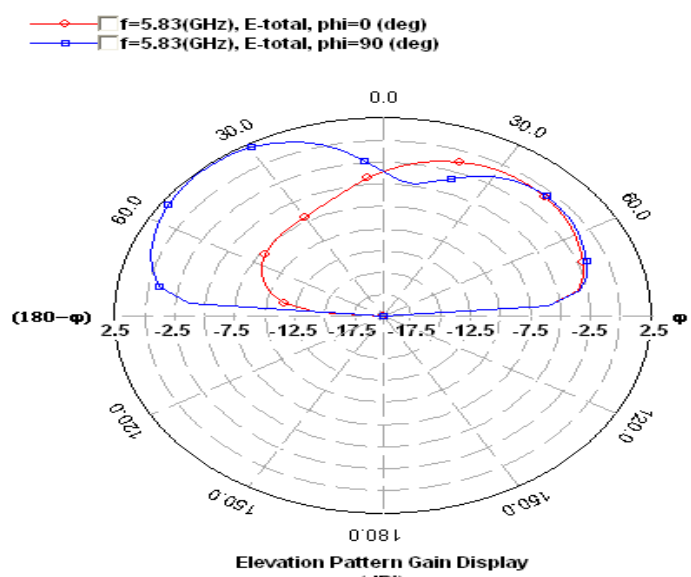

(a)

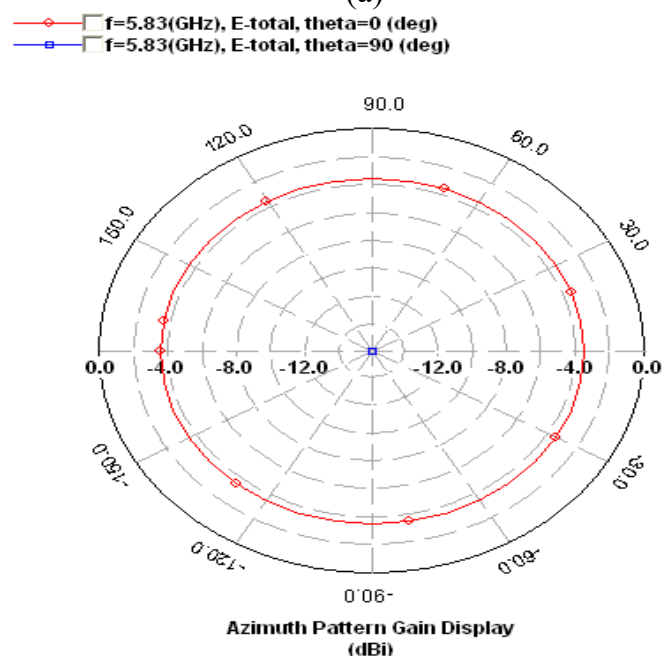

(b)

Figure 6: Radiation pattern (a) elevation (b) azimuth

Figure 7 shows the gain and directivity versus frequency plot. It is clear that proposed design shows high directivity at all resonance frequency while gain is moderate at first 3 resonance frequency. Figure 8 shows the VSWR plot (Voltage Standing Wave Ratio) of the proposed structure. For all frequency bands VSWR value is less than 2 which is within the acceptable limits for practical applications. 


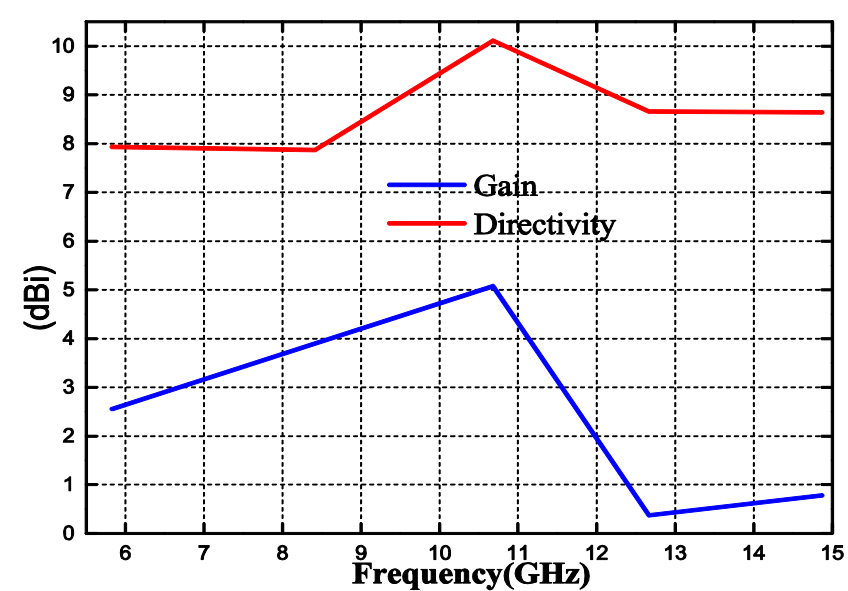

Figure 7: Gain and Directivity vs Frequency plot

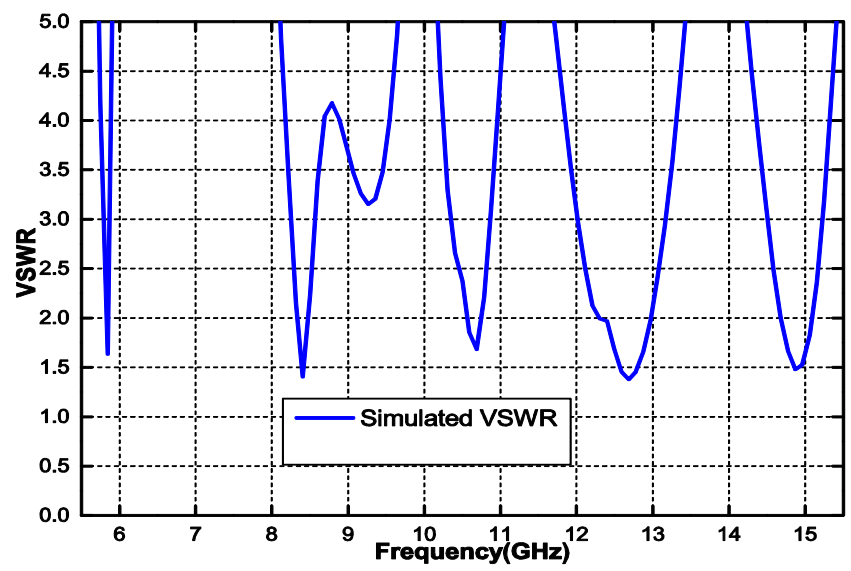

Figure 8: VSWR vs Frequency Plot

\subsection{Effective Medium Parameter Extraction of SSHSS}

Effective medium parameters of new unit cell (SSHSS) are numerically calculated by modified NRW approach formulas given in $[10,11]$. This is done by exporting the SParameters from IE3D software to MATLAB and using the following equations $[15,16]$,

$$
\begin{aligned}
& \mu_{\mathrm{r}}=\frac{2}{\mathrm{jk}_{0} \mathrm{~d}} \frac{1-\mathrm{V}_{2}}{1+\mathrm{V}_{2}} \\
& \varepsilon_{\mathrm{r}}=\frac{2}{\mathrm{jk_{0 } \mathrm { d }}} \frac{1-\mathrm{V}_{1}}{1+\mathrm{V}_{1}}
\end{aligned}
$$

Where $k_{0}$ is the wave number, $d$ is the height of the substrate. $V_{1}$ and $V_{2}$ are the composite terms which are defined in Eqs. (3) and (4) [15,16],

$$
\begin{aligned}
\mathrm{V}_{1} & =\mathrm{S}_{21}+\mathrm{S}_{11} \\
\mathrm{~V}_{2} & =\mathrm{S}_{21}-\mathrm{S}_{11}
\end{aligned}
$$

Figure 9 and Figure 10 shows the extracted permeability, permittivity and index of refraction from S-parameters for this new metamaterial structure at first two resonance frequency. From figure 9 it is clear that value of permeability, permittivity and index of refraction is negative at $5.83 \mathrm{GHz}$ frequency band.

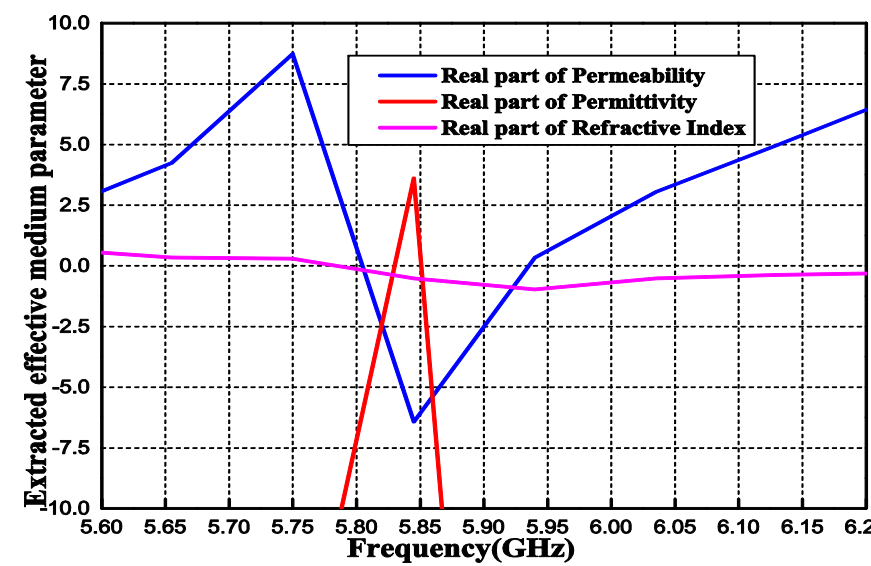

Figure 9: Extracted effective medium parameter $(6 \mathrm{GHz})$

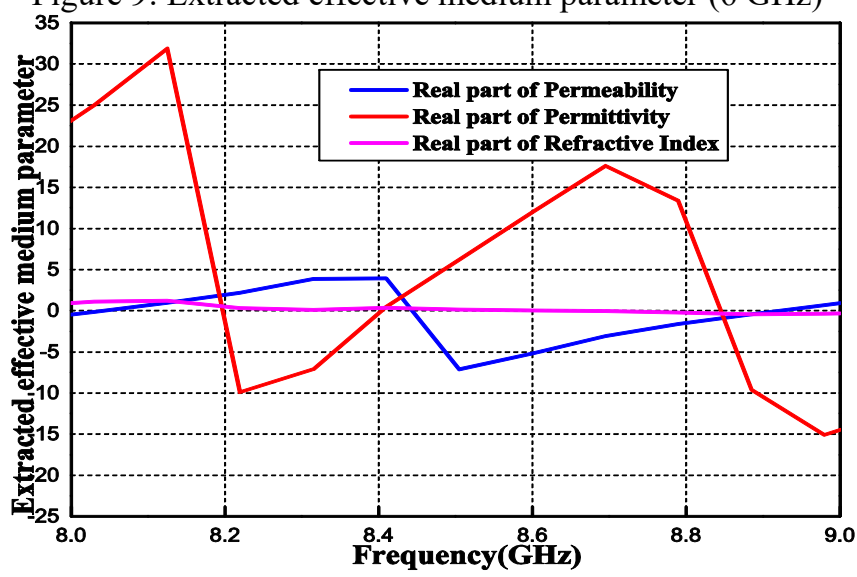

Figure 10: Extracted effective medium parameter $(8 \mathrm{GHz})$

Figure 10 depicts that value of permeability is negative, permittivity and index of refraction is near zero at $8.41 \mathrm{GHz}$.

\section{Experimental Discussion}

Figure 11 shows the top and bottom view of fabricated prototype of proposed split semi-horse shoe structure antenna (SSHSS).
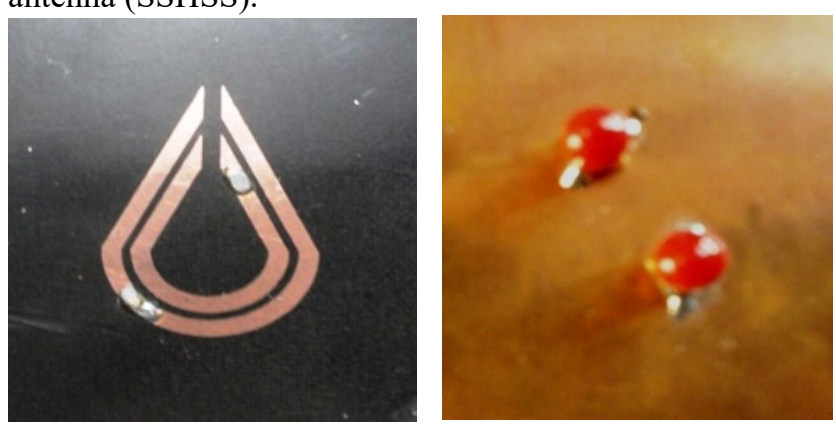

Figure 11: Fabricated prototype of proposed design

Experimental study of the fabricated prototype is done by Rohde \& Schwarz vector network analyzer (VNA) up to 20 GHz. There is a good agreement between simulated and measured results for first and fourth resonance frequency while there is small upper shift in second resonance frequency. Third band reflection coefficient gets disturbed. These changes may occur due to our limitation in fabrication. Figure 12 shows the screen shot of VNA screen. There are 3 extra band at 3.89 and $16.22,18.8 \mathrm{GHz}$ which 
were absent in simulated results. Figure 13 shows the comparison of simulated and experimental results upto 20 GHz. Figure 14 and 15 shows zoomed view of comparison between simulated and measured results for first two resonance frequencies. Summary of measured results presented in Table 3.

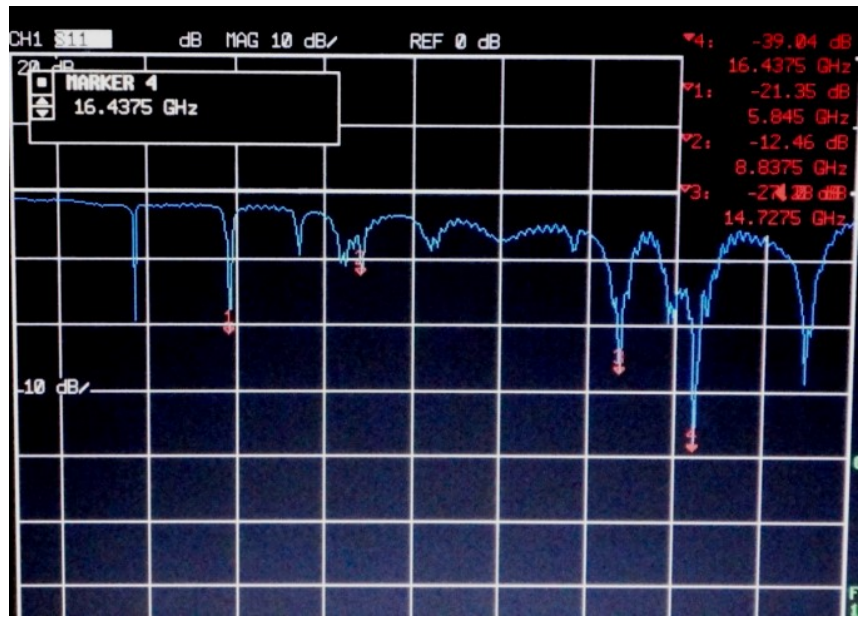

Figure 12: Measured return loss of the antenna on VNA

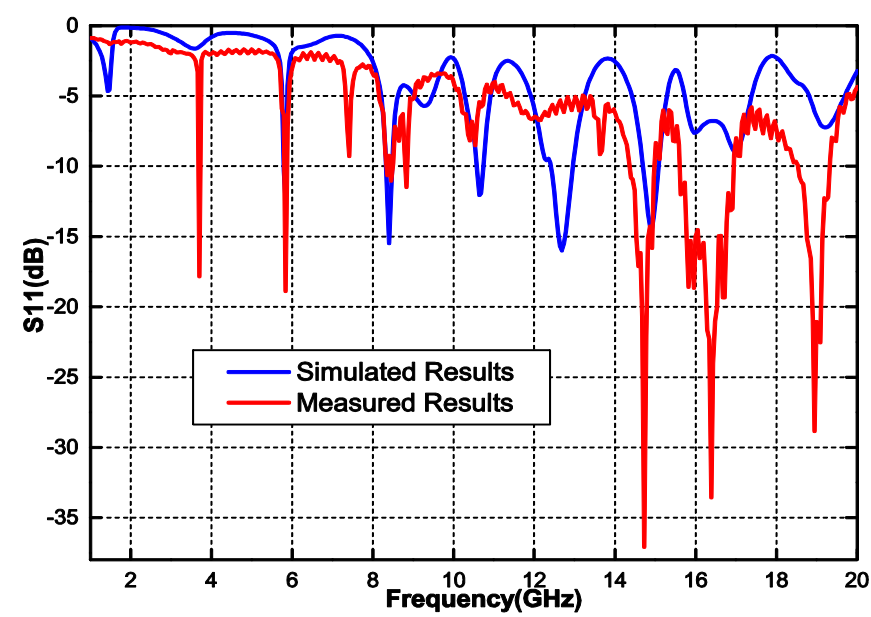

Figure 13: simulated and measured return loss

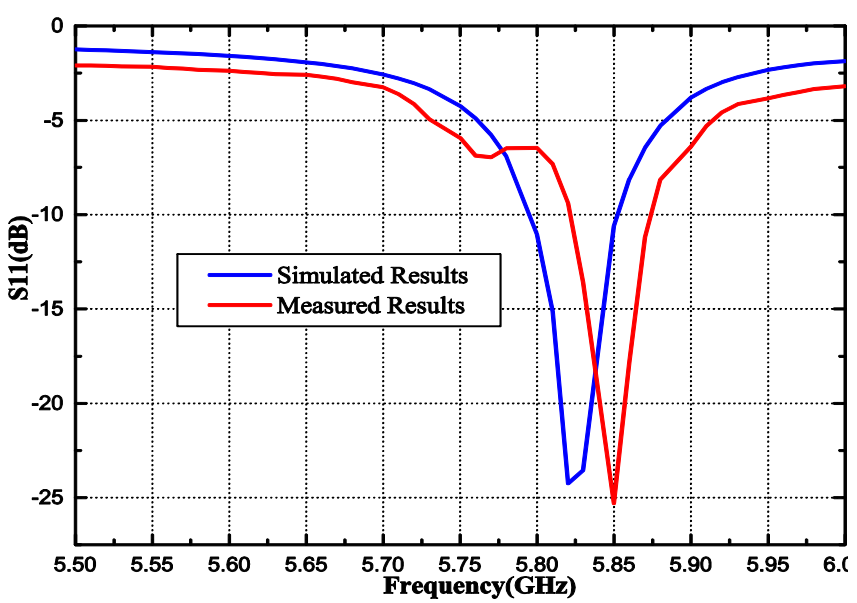

Figure 14: simulated and measured return loss at $6 \mathrm{GHz}$
Table 3 Summary of the measured results

\begin{tabular}{|c|c|}
\hline $\begin{array}{c}\text { Resonance } \\
\text { Frequency }(\mathrm{GHz})\end{array}$ & Return Loss $\mathrm{S}_{11}(\mathrm{~dB})$ \\
\hline 5.845 & -21.35 \\
\hline 8.8375 & -12.46 \\
\hline 10.5475 & -8.75 \\
\hline 13.635 & -9.14 \\
\hline 14.7275 & -27.41 \\
\hline
\end{tabular}

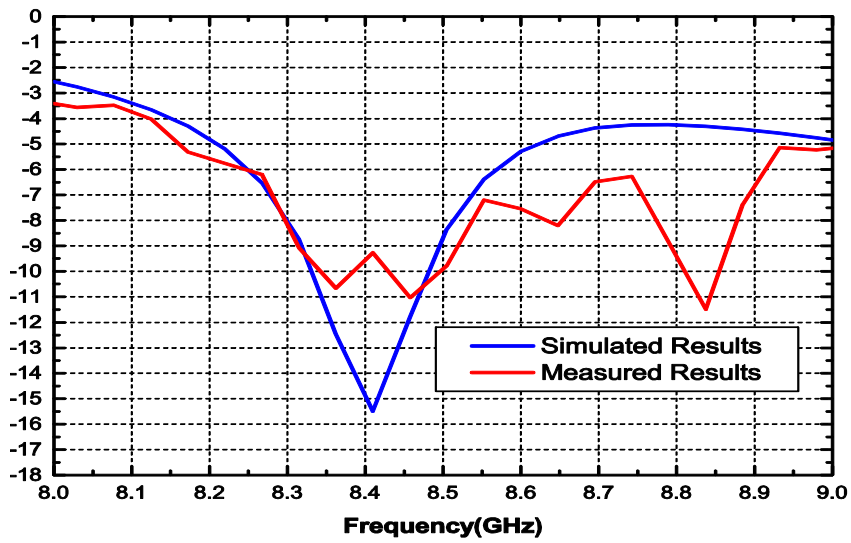

Figure 15: simulated and measured return loss at $8 \mathrm{GHz}$

\section{Conclusions}

This manuscript presents a new split semi-horse shoe structure (SSHSS) used as antenna. Theoretical analysis of the new structure done using equivalent circuit theory. This structure is planar as it does not have metallic rod on back side for getting negative permittivity. This antenna shows multiple resonance frequency due to the reactance (i.e., inductance and capacitance) present in the structure. Fabrication of new structure is simple. By changing split vertical length \& length of stub, inductance and capacitance value changed so that resonance frequency changed $\mathrm{X}$ band to ISM band in present work. Metamaterial characteristics are proved using effective medium theory at $5.83 \mathrm{GHz}$ and 8.41 GHz. It works as metamaterial inspired (ISM \& $\mathrm{X}$ band) as well as normal patch antenna. Radiation pattern, directivity and gain of the new structure were illustrated which gives the possibility of using SSHSS as the antenna. Experimental findings of antenna show close agreement with simulated \& theoretical results.

\section{References}

[1] V. G. Veselago, The electrodynamics of substances with simultaneously negative values of $\varepsilon$ and $\mu$, Soviet Physics Uspekhi, 10, 509-14, 1968.

[2] J. B. Pendry, A.J. Holden, D.J. Robbins, and W.J. Stewart, Magnetism from conductors and enhanced nonlinear phenomena,IEEE Transactions on Microwave Theory and Techniques,47(11),2075-84, 1999.

[3] D. R.Smith, W.J. Padilla, D.C. Vier, S.C. Nemat-Nasser \& S. Schultz, Composite medium with simultaneously negative permeability and permittivity, Physical Review Letters, 84(18), 4184-87, 2000. 
[4] R.W. Ziolkowski, and A. Erentok, Metamaterial-based efficient electrically small antennas, IEEE Transactions on Antennas and Propagation, 54(7), 2113-30, 2006.

[5] R. Pandeeswari and S. Raghavan, Microstrip antenna with complementary split ring resonator loaded ground plane for gain enhancement, Microwave Opt. Technol. Lett., 57(2), 292-96, 2015.

[6] Sharawi M. S., Khan M. U., Numan A. B., Aloi D. N.,A CSRR loaded MIMO antenna system for ISM band operation, IEEE Trans. Antennas Propag. , 61 (8), 4265-74, 2013.

[7] J. G. Joshi, S. S. Pattnaik, S. Devi, M.R. Lohokare and Chintakindi Vidyasagar, Offset Fed Diamond Shaped Split Ring (DSSR) Planar Metamaterial Antenna, Applied Electromagnetics Conference (AEMC-2009), 2009.

[8] Vipul Sharma, S. S. Pattnaik, Tanuj Garg and Swapna Devi, A microstrip metamaterial split ring resonator, International Journal of the Physical Sciences, 6(4), 660-63, 2011.

[9] Meenakshi Batra, S. S. Pattnaik, J.G. Joshi, Swapna Devi, Design of Planar Left-Handed Metamaterial Using Triangular Split Structure, International Symposium on Microwave and Optical Technology (ISMOT 2009), paper ID 365, 606-09, 2009.

[10] Ghanshyam Singh and S.S. Pattnaik, Metamaterial Inspired SSHSS Planar Antenna, International Journal of Advances in Microwave Technology, 3(2), 156-159, 2018.

[11] Ghanshyam Singh and S.S. Pattnaik, Metamaterial Antenna Using Split Semi-horse Shoe Structure, Advance Research in Electrical and Electronic Engineering (AREEE), 2(5), 63-67, 2015.

[12] M. F. Wu, F. Y. Meng, Q. Wu, J. Wu, L. W. Li, A Compact Equivalent Circuit Model for the SRR Structure in Metamaterials, Asia-pacific microwave conference proceedings (APMC 2005), 4-7,2005.

[13] Mohan, Sunderarajan S, The design, modeling and optimization of on-chip inductor and transformer circuits, Ph.D. Thesis, Stanford University, 38-51, 1999.

[14] Jiafu Wang, Shaobo Qu, etal, A Controllable Magnetic Metamaterial: Split-Ring Resonator with Rotated Inner Ring, IEEE Transactions on Antennas and Propagation, 56(7), 2018-22, 2008.

[15]D. R. Smith, D. C. Vier, Th. Koschny, and C.M. Soukoulis, Electromagnetic parameter retrieval from inhomogeneous metamaterials, Physical Review E,.71, pp. 1-11, 2005.

[16]Richard W. Ziolkowski, Design, Fabrication, and Testing of Double Negative Metamaterials, IEEE Transactions on Antennas and Propagation,51(7), 1516-29, 2003.

[17] IE3D user Manual, Supplied with Software. 